\title{
Prenhez ao abate em fêmeas bovinas de descarte
}

\author{
Pregnancy at slaughtered in culling bovine females \\ La preñez en hembras bovinas sacrificadas
}

\section{Resumo}

$\mathrm{O}$ aumento do abate de fêmeas é cíclico no rebanho brasileiro, normalmente influenciado por uma crise no setor, gerando uma redução nos rebanhos. $\mathrm{O}$ abate de fêmeas gestante em estágio avançado por muito tempo foi proibido, por questões sanitárias e para a manutenção dos rebanhos. O objetivo deste estudo foi caracterizar e quantificar o abate de um frigorífico na região Sul do estado do Rio Grande do Sul. Foram avaliados dados referentes ao abate de machos e fêmeas por três anos consecutivos, perfazendo o abate de 24.632 bovinos. Os dados foram classificados quanto ao ano, o mês do abate, o sexo e a prenhez (adiantada e inicial). Posteriormente, os meses do ano foram separados por quadrimestres durante todo o período de avaliação. Para a significância dos dados foi utilizado o teste do Qui-Quadrado. As fêmeas tiveram o maior índice de abate em relação aos machos, com destaque para os meses de fevereiro e novembro. Do total de fêmeas abatidas 12,69\% estavam prenhez, com maiores percentuais nos meses de março e abril. Das fêmeas gestantes, 9,99\% encontravam-se no terço final de gestação com maiores acumulados nos meses de julho a outubro. O percentual de abate de fêmeas em gestação avançada encontrado no presente estudo é alto o qual propicia perdas para o produtor diminuindo o rendimento de carcaça. Além desses fatos, o abate de vaca gestante não é aconselhável em função da questão ética e de bem-estar animal.

Palavras-chave: Abate; Ética animal; Frigorífico; Rendimento; Vaca.

\begin{abstract}
The increase in the slaughter of females is cyclical in the Brazilian herd, normally influenced by a crisis in the sector, generating a reduction in herds. The slaughter of pregnant females at an advanced stage for a long time was prohibited, for sanitary reasons and for the maintenance of herds. The objective of this study was to characterize and quantify the slaughter of a slaughterhouse in the southern region of the state of Rio Grande do Sul. Data referring to the slaughter of males and females for three consecutive years were evaluated, making up the slaughter of 24,632
\end{abstract}


cattle. Data were classified by year, month of slaughter, sex and pregnancy (advanced and early). Subsequently, the months of the year were separated by quarters throughout the evaluation period. For the significance of the data the chi-square test was used. Females had the highest rate of slaughter compared to males, especially in February and November. Of the total number of slaughtered females, $12.69 \%$ were pregnant, with the highest percentages in the months of March and April. Of the pregnant females, 9.99\% were in the final third of pregnancy with the highest accumulated in the months of July to October. The percentage of slaughter of females in advanced pregnancy found in this study is high, which causes losses for the producer, reducing carcass yield. In addition to these facts, the slaughter of pregnant cows is not advisable due to ethical and animal welfare issues.

Keywords: Animal ethics; Cow; Slaughter; Slaughterhouse; Yield.

\section{Resumen}

El aumento del sacrificio de hembras es cíclico en el rebaño brasileño, normalmente influenciado por una crisis en el sector, generando una disminución en los rebaños. El sacrificio de hembras en una etapa avanza de preñez por mucho tiempo fue prohibido, por razones sanitarias y para el mantenimiento de los rebaños. El objetivo de este estudio fue caracterizar y cuantificar el sacrificio de un matadero en la región sur del estado de Rio Grande del Sul. Se evaluaron los datos referentes al sacrificio de machos y hembras durante tres años consecutivos, que conforman el sacrificio de 24.632 vacas. Los datos se clasificaron por año, mes de sacrificio, sexo y preñez (avanzada e inicial). Posteriormente, los meses del año fueron separados por cuatrimestre a lo largo del período de evaluación. Para la significancia de los datos se utilizó la prueba de chi-cuadrado. Las hembras tuvieron la tasa de sacrificio más alta en comparación con los machos, especialmente en febrero y noviembre. Del total de hembras sacrificadas, el $12.69 \%$ estaban preñadas, con los porcentajes más altos en marzo y abril. De las hembras gestantes, el 9,99\% se encontraba en el último tercio de gestación con el mayor número en los meses de julio a octubre. El porcentaje de sacrificio de hembras en gestación avanzada encontrado en este estudio es alto, lo que conlleva pérdidas para el productor, ya que reduce el rendimiento en canal y, en consecuencia, genera pérdidas para los mataderos. Además de estos hechos, el sacrificio de vacas gestantes no es aconsejable por cuestiones éticas y de bienestar animal.

Palabras clave: Ética animal; Planta de sacrificio; Rendimiento; Sacrificio; Vaca.

\section{Introdução}

A bovinocultura de corte é uma importante atividade econômica no Brasil. O país detém o maior rebanho comercial do mundo, dispondo de 187,55 milhões de cabeças, abatendo no ano de 2020 cerca de 41,5 milhões de cabeças (ABIEC, 2021), sendo parte deste montante advinda do abate de fêmeas bovinas.

$\mathrm{Na}$ última década o abate de fêmeas bovinas no Brasil variou entre 40 a 50\% (ANUALPEC, 2018; IBGE, 2021), sendo este percentual alterado de acordo com os estados da federação e impulsionado em momentos de crise econômica do setor. Nesse sentido, a comercialização de fêmeas de descarte é uma das importantes fontes de receita dos sistemas produtivos de ciclo completo (Missio et al., 2013; Gonçalves et al., 2017; Vaz et al., 2020), devido à similaridade do peso de abate e de carcaça em relação aos novilhos (Pacheco et al., 2013). Outro reflexo desse abate, deriva em alguns momentos da melhoria das taxas reprodutivas dos rebanhos (Ferreira et al., 2013; Torres; Tineo; Raidan, 2015), bem como, a redução da idade ao primeiro acasalamento (Vaz et al., 2012b; Klein et al., 2021) e o aumento na taxa de reposição de fêmeas impulsionada pela maior pressão de seleção do sistema produtivo (Borba et al., 2011).

Embora seja expressivo o abate de fêmeas bovinas, a idade cronológica das mesmas é avançada, quando comparada aos machos (Vaz et al., 2021), sendo classificadas como vacas de descarte, e comercializadas recebendo preços distintos das demais (Pacheco et al., 2013). O menor valor pago pelas fêmeas na comercialização para abate se deve a maior idade, pela maior expressão de temperamento, pela menor qualidade de carne (Missio et al., 2013) e pela ampla variação no rendimento de carcaça (Pascoal et al., 2011; Vaz et al., 2012a), muito em função da quantidade de contusões nas carcaças (Mendonça et al., 2017; Bethancourt-Garcia et al., 2019). Neste sentido, tanto o produtor rural quanto a indústria perdem nesta comercialização (Missio et al., 2013).

O abate de vacas prenhes em estágio avançado de gestação, tem sido abordado globalmente (Riehn et al., 2011; Nielsen et al., 2019; Adebowale; Ekundayo; Awoseyi, 2020; Njoga et al., 2021; Zitterer; Paulsen, 2021) relatando principalmente o desperdício fetal, evidenciando ser este um problema relacionado ao bem-estar animal (Ademola, 2010). 
São inúmeros os fatores que contribuem para o envio de fêmeas gestantes para o abate. É comum os relatos bibliográficos mostrando problemas de baixa produção de leite (Zitterer; Paulsen, 2021), presença de doenças (Merchioratto et al., 2020), e diagnóstico de prenhez com resultado falso-negativo (Nielsen et al., 2019). No Brasil, Sornas et al. (2014) ao avaliarem o abate de fêmeas em avançado estágio de estação, verificaram ser comum os produtores enviarem fêmeas para o abate, sem prévio diagnóstico gestacional.

De acordo com a Organização Mundial de Saúde Animal - OIE (2016), fêmeas no terço final de gestação não devem ser transportadas em caminhões e nem abatidas, tais ações ferem os preceitos de bem-estar animal, tendo em vista que os estresses sofridos pelos animais nessas operações podem ocasionar contusões, dores e abortos (Mendonça et al., 2018; Bethancourt-Garcia et al., 2019; Oliveira; Tenedini; Ribeiro 2021). Nessa perspectiva, o transporte de vacas prenhes deve ser realizado somente em momentos de extrema necessidade. Outro aspecto está relacionado ao feto, existem evidências bibliográficas de que fetos de mamíferos são sencientes a partir da segunda metade da gestação (Bellieni; Buonocore, 2012). Por razões de ética e bem-estar animal os países da União Europeia, condenam e proíbem o transporte de fêmeas estando com 90\% do período de gestação. Na Alemanha, por exemplo, o abate de fêmeas no terço final de gestação é rigorosamente proibido (Zitterer, Paulsen, 2021). Entretanto no Brasil tais práticas ocorrem sem que existam multas ou penalidades.

Desta forma, o objetivo deste estudo foi caracterizar e quantificar o abate de um frigorífico na região Sul do estado do Rio Grande do Sul que opera sobre inspeção estadual.

\section{Metodologia}

O presente trabalho é uma pesquisa de campo de aspecto quantitativo (Pereira et al., 2018), a qual foi realizada em propriedade particular, tendo como referencial teórico para discussão dos resultados livros, dissertações, teses e artigos científicos de diferentes periódicos.

O trabalho foi conduzido em um frigorífico localizado na região Sul do Rio Grande do Sul, que opera sobre os critérios de inspeção estadual. Foram avaliados os dados referentes ao abate de machos e fêmeas por três anos consecutivos, perfazendo o abate de 24.632 bovinos. Os dados foram disponibilizados em um arquivo eletrônico (planilha do Excel) contendo as informações referentes ao ano/mês, lote de origem, local de origem, número de animais, número de abates, sexo, prenhez e condenações.

Os dados foram classificados quanto ao ano, o mês do abate, o sexo (machos e fêmeas) e a prenhez (adiantada e inicial). Os meses do ano foram separados por quadrimestres durante todo o período de avaliação, ficando da seguinte forma:

Quadrimestre 1 - outubro, novembro, dezembro e janeiro; Quadrimestre 2 - fevereiro, março, abril e maio e Quadrimestre 3 - junho, julho, agosto e setembro.

Foram enviados para o abate durante o período de estudo 3.604 lotes de distintas regiões do Estado do RS, com média mensal de 700 bovinos abatidos. Dos 24.632 animais abatidos, 12.779 (51,88\%) foram fêmeas e 11.853 (48,12\%) machos.

Os dados brutos foram organizados em planilhas e analisados por frequências das ocorrências. As frequências foram obtidas a partir de dados agrupados durante os 3 anos mensalmente, depois por quadrimestre. Para a significância dos dados $(\mathrm{P}<0,05)$ foi utilizado o teste do Qui-Quadrado.

\section{Resultados e Discussão}

As fêmeas apresentaram maior índice de abate em comparação aos machos ao longo dos anos destacando-se os meses de fevereiro e novembro com média de 57,72\% (Gráfico 1). Entretanto, os meses de maio e junho se destacaram pela maior proporção no abate de machos perfazendo $58,74 \%$ e $59,21 \%$, respectivamente, do total de animais abatidos. 
Gráfico 1 - Relação de abate de machos e fêmeas nos diferentes anos.

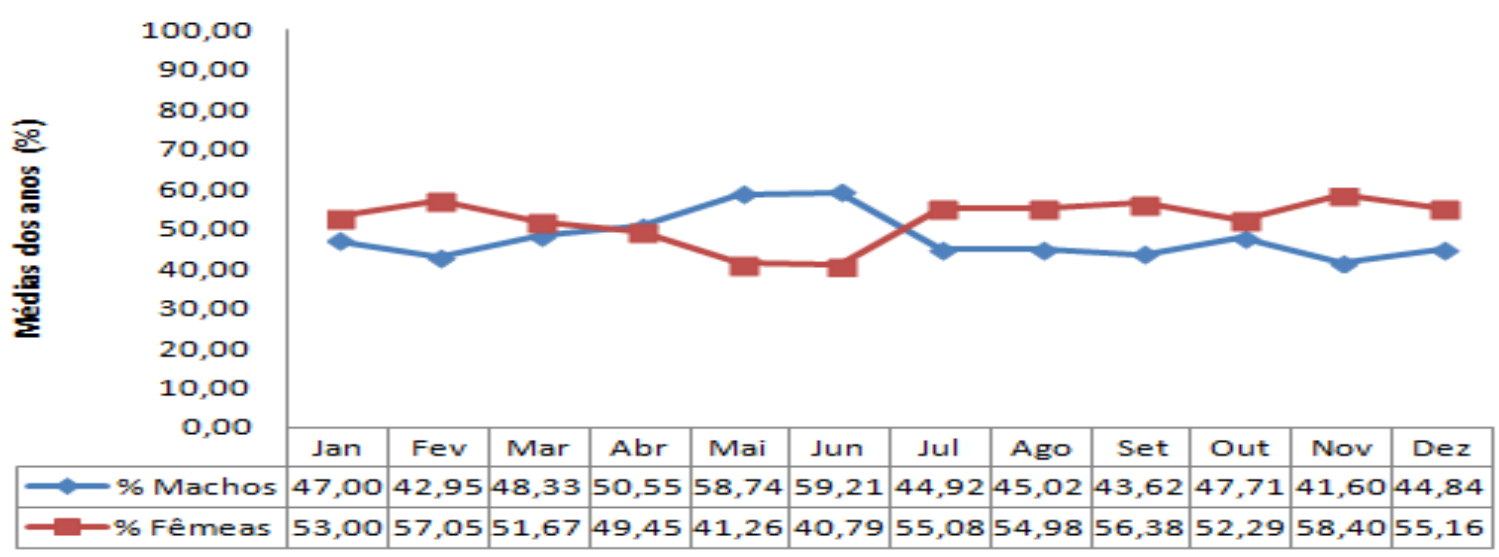

Fonte: Autores.

O total de fêmeas abatidas mensalmente, média de três anos, mostra o abate de fêmeas com oscilações durante os meses do ano (Gráfico 2).

Gráfico 2 - Distribuição do abate médio de fêmeas nos diferentes meses do ano.

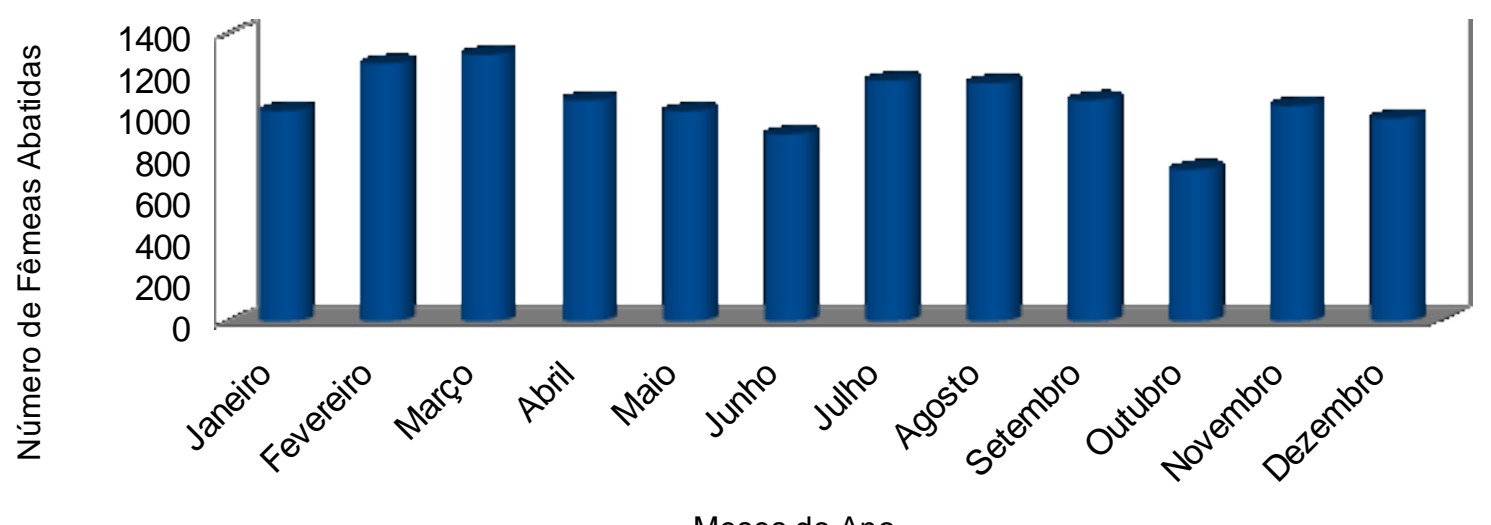

Meses do Ano

Fonte: Autores.

As maiores quantidades de fêmeas abatidas, média dos três anos, foram nos meses de março e fevereiro, perfazendo 1.298 e 1.255 animais, respectivamente. Por outro lado, no mês de outubro ocorreu menor incidência de abate de fêmeas, com 741 fêmeas na média nos três anos.

O percentual de fêmeas prenhes abatidas no frigorífico em estudo é alto, perfazendo 12,69\% do total de fêmeas encaminhadas ao abate (12.779). Os meses de março e abril apresentaram maior incidência de vacas prenhes com $22,8 \%$ e 20,63\%, respectivamente (Tabela 1). Por outro lado, nos meses de julho a outubro houve menor incidência de prenhez, sendo em média de $6,15 \%$ de fêmeas gestantes, pois é o período próximo ao parto em propriedades que utilizam a estação de monta tradicional. 
Tabela 1 - Número de vacas não prenhes e prenhes e a frequência mensal de prenhez em relação ao total de fêmeas abatidas.

\begin{tabular}{lccc}
\hline Meses & Não Prenhe & Prenhe & Prenhe (\%) \\
\hline Janeiro & 909 & 118 & $11,31^{\mathrm{CD}}$ \\
Fevereiro & 1113 & 142 & $11,31^{\mathrm{CD}}$ \\
Março & 987 & 311 & $24,00^{\mathrm{A}}$ \\
Abril & 845 & 231 & $21,47^{\mathrm{A}}$ \\
Maio & 872 & 153 & $14,84^{\mathrm{B}}$ \\
Junho & 815 & 97 & $10,64^{\mathrm{D}}$ \\
Julho & 1080 & 92 & $7,85^{\mathrm{E}}$ \\
Agosto & 1064 & 95 & $8,20^{\mathrm{E}}$ \\
Setembro & 993 & 85 & $7,88^{\mathrm{E}}$ \\
Outubro & 694 & 47 & $6,34^{\mathrm{F}}$ \\
Novembro & 926 & 124 & $11,81^{\mathrm{CD}}$ \\
Dezembro & 859 & 130 & $13,14^{\mathrm{B}}$ \\
\hline Total & 12779 & 1622 & 12,69 \\
\hline
\end{tabular}

ABCDEF Teste qui-quadrado $(\mathrm{P}<0,05)$. Fonte: Autores.

No frigorífico em estudo, a ocorrência de fêmeas em estágio avançado de prenhez (terço final de gestação) determina o destino das mesmas como graxaria industrial. No terço final da gestação foram contabilizadas 162 fêmeas, correspondendo a $9,99 \%$ do total de fêmeas prenhes destinadas ao abate (1622). O período compreendido entre agosto e outubro foi o de maior incidência de fêmeas no terço final de gestação (Gráfico 3), com total de 27,65\% de prenhez adiantada em relação ao total de vacas prenhes no mês de outubro. As vacas com gestação adiantada abatidas durante o período de avaliação do presente estudo foram $12,25 \%$ do total de vacas prenhes abatidas e $1,28 \%$ do total de vacas abatidas.

Gráfico 3 - Incidência média mensal de fêmeas prenhes no terço final da gestação.

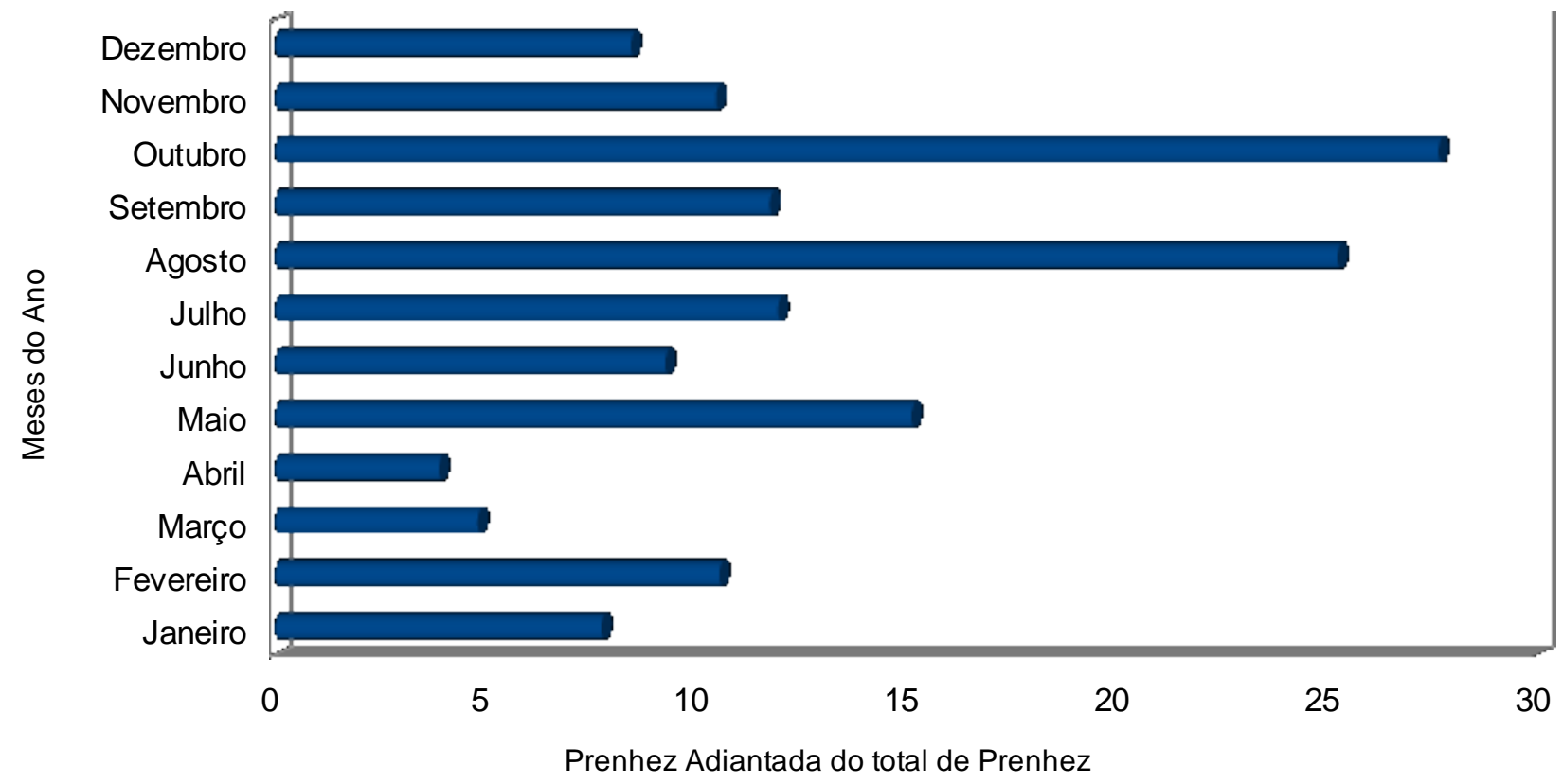

Fonte: Autores. 
O quadrimestre composto pelos meses de fevereiro, março, abril e maio teve menor incidência, com 7,41\% das vacas prenhes em estádio de gestação adiantada, enquanto o quadrimestre formado pelos meses de junho, julho, agosto e setembro prefez 14,63\% de vacas abatidas em estádio de gestação adiantada (Tabela 2).

Tabela 2 - Número de vacas prenhes, prenhez adiantada e frequência de vacas no terço final da gestação de acordo com o quadrimestre.

\begin{tabular}{lccc}
\hline \multicolumn{1}{c}{ Quadrimestre } & Prenhe, N & Terço Final da Gestação, N & Terço Final (\%) \\
\hline 1 (Outubro, Novembro, Dezembro e Janeiro) & 371 & 46 & $11,03^{\mathrm{A}}$ \\
2 (Fevereiro, Março, Abril e Maio) & 774 & 62 & $7,41^{\mathrm{B}}$ \\
3 (Junho, Julho, Agosto e Setembro) & 315 & 54 & $14,63^{\mathrm{A}}$ \\
\hline Total & 1460 & 162 & 9,99 \\
\hline
\end{tabular}

${ }^{\mathrm{AB}}$ Teste qui-quadrado $(\mathrm{P}<0,05)$. Fonte: Autores.

\section{Discussão}

A relação de animais abatidos, bem como o sexo de maior frequência destinado ao abate no presente estudo, corresponde ao andamento da pecuária de corte na região, bem como a exigência do mercado. O maior número de fêmeas é em função de atender o mercado consumidor da indústria, o qual não exige abate de novilhos.

No Brasil, de acordo com dados do IBGE (2021), entre os anos de 2017 a 2020 o percentual de abate de fêmeas no país variou entre 40,6 e 42,1\%. Entretanto, no estado do Rio Grande do Sul de acordo com os dados da Secretaria da Agricultura, Pecuária e Desenvolvimento Rural (Rio Grande Do Sul, 2020), desde o ano de 2017 até 2019 o abate de fêmeas foi superior a 50\%, fato que corrobora com o resultado obtido nesse estudo. O abate maior de 50\% de fêmeas ocasiona a redução dos rebanhos, normalmente verificada nos momentos de crise do setor (Sornas et al., 2014). O aumento do abate de fêmeas pode ser devido à implantação de novas tecnologias, intensificando os sistemas de produção, proporcionando melhores índices produtivos, aumentando a taxa de descarte dos rebanhos (Ferreira et al., 2013; Torres et al., 2015). Visto que, a taxa de reposição de um rebanho estável gira em torno de 20\%, com taxas de desfrute superiores, o índice de seleção é mais intenso, fazendo o descarte aumentar, e consequentemente, há o aumento de fêmeas destinadas ao abate (Mello et al., 2013).

A vaca de descarte apesar de possuir qualidade inferior e baixos rendimentos (Dick, et al., 2019), é um produto necessário para o abastecimento do mercado interno, em que os frigoríficos em muitas situações têm preferência por esse tipo de produto (Missio et al., 2013), pois ganham maior margem de lucro, pela diferenciação de preço pago ao produtor em relação ao sexo e as categorias abatidas (Vaz et al., 2012a). Vaz et al., (2021) ao analisarem a agregação de valor em bovinos destinados a programa de certificação de Carne Angus no Sul do Brasil, verificaram ser o percentual de certificação de fêmeas menor em relação aos machos, em função da maior idade dessas fêmeas, e consequentemente, a desclassificação do programa.

A maior concentração de abates de fêmeas nos meses de fevereiro e março está associada à maior oferta qualitativa e quantitativa de forragem de baixo custo de produção para terminação dos animais (Ferreira et al., 2011; Mezzalira et al., 2012). O elevado abate de fêmeas no primeiro trimestre é considerado normal, pois corresponde ao período de descarte de vacas que não emprenharam na estação de monta planejada ou tradicional, sendo esse período iniciado em outubro do ano anterior (Knapik, et al., 2011). Mesmo não prenha, até o início do outono ocorre crescimento e melhoria na qualidade das pastagens naturais, se mantendo em determinados anos até a metade do outono, podendo ocorrer a terminação das vacas com baixo custo de produção, com o final desse período coincidindo com o diagnóstico de gestação das fêmeas (Vaz et al., 2021). Nos sistemas de produção, as vacas por ocasião do diagnóstico de gestação podem permanecer na propriedade ou serem encaminhadas ao 
abate, ações que dependerão do nível tecnológico empregado ao sistema. O fato de manter as vacas na propriedade tem como finalidade fornecer condições para aumentar as chances desse indivíduo emprenhar na estação de monta subsequente, no entanto, tal estratégia é vista como improdutiva, pois, é fundamental que a vaca deixe um bezerro anualmente na propriedade (Gonçalves et al., 2017; Vaz et al., 2020). Nesse sentido, fêmeas com o parecer negativo de prenhez, após o diagnóstico de gestação normalmente formarão lotes de descarte na propriedade, com isso, vacas que apresentam bom estado de condição corporal são enviadas ao abate, e no caso de estarem magras são submetidas a terminação, ainda em pastagens naturais ou pastagens de inverno. Para os sistemas de produção é importante a comercialização imediata das vacas de descarte, logo após o diagnóstico de gestação, o que propicia liberação de áreas e melhor disponibilidade de pasto para as demais categorias (Vaz et al., 2012a).

O fato dessas vacas não emprenharem na estação de monta e serem descartadas pode estar relacionado pelo déficit energético sofrido nesse período, ou seja, o animal não consegue ingerir o alimento suficiente para manter suas funções reprodutivas (Zago; Canozzi; Barcellos, 2019), ou ainda pela ocorrência de problemas sanitários (Alfieri; Alfieri, 2017; Lopes et al., 2017). Convém salientar, ter o aumento de fêmeas abatidas reflexos no mercado de bezerros dos próximos anos, devido o menor número de nascimentos de bezerros (Gonçalves et al., 2017).

A maior percentagem de animais prenhes abatidas nos meses de março e abril coincide com o maior número de fêmeas abatidas. Um aspecto importante que deve ser considerado nesses meses deriva da necessidade do produtor gerar receita com estes animais antevendo o período de diminuição da qualidade da forragem (Pascoal, et al., 2011), e principalmente, para evitar o período de escassez forrageira no inverno. Vacas com idade cronológica avançada apresentam maiores problemas de dentição e, se permanecerem durante o inverno na propriedade, perderão em desempenho e eficiência produtiva.

A prenhez das vacas causa prejuízos tanto para o produtor rural, quanto para o frigorífico (Oliveira et al., 2021), além de contribuir na diminuição dos rebanhos de bovinos da região. Essa ocorrência pode ser consequência do período de acasalamento, pois a estação de monta no Rio Grande do Sul para o gado de corte, na maioria dos casos é de maneira tradicional, ou seja, no período entre primavera e verão.

Após a estação de monta é necessário que se faça a correta detecção de prenhez, se positivo as fêmeas seguirão na cria, caso contrário serão destinadas ao descarte. Porém, em alguns casos essa detecção de prenhez pode ser falha e essas vacas destinadas ao descarte podem estar prenhas, ocasionando prejuízos ao frigorífico e ao produtor. Outro fator de ocorrência de prenhez ao abate está atrelado a vacas que supostamente não emprenharam na estação reprodutiva anterior, mas durante a fase de terminação foram propositalmente emprenhadas para um teórico ganho de peso mais acelerado quando comparado ao de vacas não gestantes (Grassi; Müller, 1991). A ideia da prenhez se dá em função de evitar a ocorrência dos ciclos estrais normais das vacas, as quais, durante o período de cio mudam o comportamento diminuindo o consumo de alimento e se movimentando mais na busca do acasalamento. Além desse fato, vacas prenhes ou castradas tendem a ter melhor acabamento de carcaça (Silva et al., 2006). Nos dias de cio, com montas sucessivas ocorrendo entre animais nesta fase, podem gerar contusões nos animais. Essas contusões, quando ocasionadas próximo do abate podem ocasionar perdas consideráveis de músculos por ocasião da toilette dos animais ao abate (Mendonça et al., 2018; Bethancourt-Garcia et al., 2019). No entanto, emprenhar a vaca para que durante a terminação não ocorram cios, pode não ser econômico aos sistemas de produção, pois ao gestar, as suas exigências nutricionais são aumentadas e o provável maior ganho de peso pode estar associado também a um maior consumo de alimentos, sendo esse aumentado com o avanço da gestação (NRC, 2016).

Outra opção buscada pelos produtores para a não ocorrência de cios durante a terminação das vacas é a ovariectomia (De Paiva Carvalho et al., 2010), a qual consta da retirada total dos ovários, impedindo assim os ciclos estrais normais das vacas. Ambas as situações são causadoras de traumas as vacas e os seus resultados são controversos na literatura, com 
resultados positivos (Chacur et al., 2007) e ou negativos (Meirelles et al., 2007; De Paiva Carvalho et al., 2010). As técnicas de ovariectomia e de emprenhar as vacas para melhor comportamento na terminação, as quais foram utilizadas pelos produtores, são penalizadas pelos frigoríficos, pois as mesmas causam perdas aos produtores e a indústria a qual não consegue o volume de mercadoria necessária para atender os mercados consumidores.

A incidência de vacas prenhes no final da gestação com a condenação das carcaças foi maior no quadrimestre formado pelos meses de junho, julho, agosto e setembro, por coincidir com a proximidade dos partos dos sistemas produtivos que visam a produção de bezerros nascidos na primavera. Isso pode ocorrer devido à dificuldade que o criador encontra em assimilar a gestação de vacas, visto que, varia de acordo com o peso, tamanho, raça, alimentação do animal, dentre outros. Além da gestação, o produtor é penalizado, visto que há diminuição do rendimento da carcaça à medida que a gestação progride, pois o peso vivo é mais elevado em decorrência dos pesos do feto, útero e membranas fetais, aproximando $82 \mathrm{~kg}$ no final da gestação.

De acordo com o Regulamento da Inspeção Industrial e Sanitária de Produtos de Origem Animal RIISPOA publicado no ano de 1952 (revogado), deve ser evitado o abate de fêmeas em estado adiantado de gestação, ou seja, terço final, (a partir de 6 meses ou $55 \mathrm{~cm}$ de comprimento) e ao estar nesse estágio avançado era permitido somente o aproveitamento condicional pelo frigorífico da carcaça, sendo destinado a graxaria (condenado) ou carne enlatada. Porém, essa prática foi flexibilizada pelo RIISPOA (Brasil, 2017) em sua atualização, o qual libera o abate com o aproveitamento da carcaça pelo frigorífico, somente fazendo o desconto do peso do feto. Concomitante a isso, no estado do RS há a lei no 11.915/2003 que em seu Art. 13 proíbe o transporte de fêmeas em adiantado estado de gestação, e no decreto n53.848/2017 em seu Art. 215, constitui como infração o abate de fẽmeas em adiantado estado de gestação, mas não há previsão de penalidades para o infrator. $\mathrm{O}$ abate de fêmeas gestantes além de cruel, por outro lado oferece riscos à saúde das pessoas, tanto aos funcionários dos frigoríficos, quanto de quem se alimenta desses produtos (Mufinda; Boinas; Nunes, 2017). Isso se deve ao fato de que vacas prenhes são mais suscetíveis à infecção pela bactéria que causa a brucelose, doença que pode afetar o sistema nervoso central, o coração, os ossos, as articulações, o fígado e o aparelho digestivo. A transmissão se faz por contaminação direta pelo contato com fetos abortados, placentas e descargas uterinas, ou pelo consumo de carne e laticínios (Lawinsky et al., 2010; Carvalho et al., 2016). Em termos de bem-estar animal existe também no momento do abate a preocupação com o feto, de acordo com a orientação da Organização Mundial de Saúde Animal - OIE (2016) o feto não pode ser removido do útero antes de 5 minutos após a sangria da fêmea, a fim de que não venha a recobrar consciência. Neste período, ainda há batimentos cardíacos fetais, bem como, movimentos. Caso o feto seja retirado do útero antes do período recomendado e venha a respirar este deve ser morto por meio de um dardo cativo ou equipamento semelhante.

\section{Conclusão}

O aumento da frequência de abate de fêmeas gera redução nos rebanhos, ora por intensificação, ora por crise no setor. O percentual de abate de fêmeas em gestação avançada encontrado no presente estudo é alto, o qual propicia perdas para o produtor, uma vez que diminui o rendimento de carcaça e, consequentemente, gera perda também para as indústrias frigoríficas.

As pesquisas futuras relacionadas ao assunto além da caracterização e da contabilização das prenhez podem procurar calcular efetivamente as perdas através de pesagens e, também devem ver a influência da prenhez na qualidade sensorial da carne de vacas bovinas abatidas nos frigoríficos. 


\section{Referências}

ABIEC - Associação Brasileira das Indústrias Exportadoras de carne (Ed.). Perfil da Pecuária no Brasil: Relatório Anual, 2021. Abiec.

Adebowale, O. O., Ekundayo, O., \& Awoseyi, A. A. (2020). Female cattle slaughter and foetal wastage: A case study of the Lafenwa abattoir, Ogun state, Nigeria. Cogent Food \& Agriculture, 6(1), 1809308.

Ademola, A. I. (2010). Incidência de desperdício fetal em gado abatido no matadouro e covil Oko-Oba, Agege, Lagos, Nigéria. Veterinary Research (Pakistan), 3(3), 54-57.

Alfieri, A. A., \& Alfieri, A. F. (2017). Doenças infecciosas que impactam a reprodução de bovinos. Revista Brasileira de Reprodução Animal, 41(1), 133-139.

ANUALPEC - Anuário estatístico da produção animal. Prol Editora Gráfica, 2018. 350 p.

Bellieni, C. V., \& Buonocore, G. (2012). Is fetal pain a real evidence? The Journal of Maternal-Fetal \& Neonatal Medicine, 25(8), 1203-1208.

Bethancourt-Garcia, J. A., Vaz, R. Z., Vaz, F. N., Silva, W. B., Pascoal, L. L., Mendonça, F. S., Silva, W. B., Pascoal, L. L., Mendonça, F. S., Vara, C. C., Nuñez, A. J. C., Restle, J. (2019). Pre-slaughter factors affecting the incidence of severe bruising in cattle carcasses. Livestock Science, 222(1), 41-48.

Borba, L. H. F., Rey, F. S. B., Silva, L. O. C. D., Boligon, A. A., \& Alencar, M. M. D. (2011). Parâmetros genéticos para características de crescimento e reprodução de bovinos da raça Canchim. Pesquisa Agropecuária Brasileira, 46(11), 1570-1578.

Brasil. DECRETO No 9.013, DE 29 DE MARÇO DE 2017. Altera Lei $\mathrm{n}^{\circ} 1.283$, de 18 de dezembro de 1950, e a Lei $\mathrm{n}^{\circ}$ 7.889, de 23 de novembro de 1989. Aprova o regulamento da Inspeção Industrial e Sanitária de Produtos de Origem Animal (RIISPOA). Diário Oficial da União, Brasília, DF. 62, 3 de 29 de março de 2017

Carvalho, R. F. B. D., Santos, H. P., Mathias, L. A., Pereira, H. D. M., Paixão, A. P., Costa, V. M., \& Alves, L. M. C. (2016). Frequência de brucelose bovina em rebanhos leiteiros e em seres humanos na região central do estado do Maranhão, Brasil. Arquivos do Instituto Biológico, 83(1-6), e1042014.

Chacur, M. G. M., de Faria Marin, M., Oba, E., \& do Nascimento Kronka, S. (2007). Efeito da ovariectomia em novilhas Nelore x Angus, Bos taurus indicus x Bos taurus taurus sobre os ganhos de peso. Semina: Ciências Agrárias, 28(2), 317-322.

De Paiva Carvalho, C. R., Marcon, C. C., Kozicki, L. E., Junior, C. F. B., \& da Costa, C. E. M. P. (2010). Ovariectomia visando à engorda de vacas de corte em confinamento. Revista Acadêmica Ciência Animal, 8(4), 405-408.

Dick, M., da Silva, M. A., de Souza, J. P., da Silva, R. R. F., Ferreira, O. G. L., \& de Souza Maia, M. (2019). Impactos econômicos da condenação de carcaças na produção de bovinos no Sul do Brasil. Archivos Latinoamericanos de Producción Animal, 27(1-4), 33-37.

Ferreira, E. T., Nabinger, C., Elejalde, D. A. G., Freitas, A. K. D., Schmitt, F., \& Tarouco, J. U. (2011). Terminação de novilhos de corte Angus e mestiços em pastagem natural na região da Campanha do RS. Revista brasileira de zootecnia, 40, 2048-2057.

Ferreira, M. C. N., Miranda, R., Figueiredo, M. A., Costa, O. M., \& Palhano, H. B. (2013). Impacto da condição corporal sobre a taxa de prenhez de vacas da raça nelore sob regime de pasto em programa de inseminação artificial em tempo fixo (IATF). Semina: Ciências Agrárias, 34(4), 1861-1868.

Gonçalves, G. V. B., Vaz, R. Z., Vaz, F. N., Mendonça, F. S., Fontoura, J. A. S. D., \& Castilho, E. M. (2017). Análise de custos, receitas e ponto de equilíbrio dos sistemas de produção de bezerros no rio grande do sul. Ciência Animal Brasileira, 18(1), e46329.

Grassi, C., \& Müller, L. (1991). Efeito do manejo de vacas de descarte no desempenho e nas características da carcaça. Pesquisa Agropecuária Brasileira, 26(8), 1175-1181.

IBGE - Instituto Brasileiro de Geografia e Estatística. Estatística da Produção Pecuária Primeiros resultados jan.-mar. 2021. https://biblioteca.ibge.gov.br/visualizacao/periodicos/3087/epp_pr_2021_1tri.pdf

Klein, J. L., Adams, S. M., Alves Filho, D. C., Brondani, I. L., Pizutti, L. Â. D., Antunes, D. P., \& da Silva Mello, D. A. (2021). Programação fetal e as consequências no desenvolvimento da progênie-uma revisão. Research, Society and Development, 10(12), e557101220766.

Knapik, K., Kozicki, L. E., Segui, M. S., Pereira, A., dos Santos Breda, J. C., Pimpão, C. T., Weiss, R. R., \& dos Santos, I. W. (2011). Desempenho reprodutivo de bovinos de corte da raça Brangus e sua relação com os meses da estação de parturição no Sul brasileiro. Revista Acadêmica Ciência Animal, 9(3), 263-269.

Lawinsky, M. L. D. J., Ohara, P. M., Elkhoury, M. D. R., Faria, N. D. C., \& Cavalcante, K. R. L. J. (2010). Estado da arte da brucelose em humanos. Revista Pan-Amazônica de Saúde, 1(4), 75-84.

Lopes, L. B., Rodrigues, R. O., Eckstein, C., \& Moustacas, V. S. (2017). Serological profile of bovine leptospirosis in cows slaughtered in slaughterhouse of ColÃder, Mato Grosso. Scientific Electronic Archives, 10(3), 29-34.

Mufinda, F. C., Boinas, F., \& Nunes, C. (2017). Prevalência e factores associados à brucelose humana em profissionais da pecuária. Revista de Saúde Pública, 51(1) 1-8.

Meirelles, C., Junior, C. F. B., Kozicki, L. E., Weiss, R. R., \& Segui, M. S. (2007). Avaliação do ganho de peso de novilhas ovariectomizadas por técnica transvaginal. Revista Acadêmica Ciência Animal, 5(3), 303-307.

Mello, J. C. C. B. S. D., Gomes, E. G., Abreu, U. G. P. D., Carvalho, T. B. D., \& Zen, S. D. (2013). Análise de desempenho de sistemas de produção modais de pecuária de cria no Brasil. Production, 23(4), 877-886. 
Mendonça, F. S., Vaz, R. Z., Cardoso, F. F., Restle, J., Vaz, F. N., Pascoal, L. L., Reimann, F. A., \& Boligon, A. A. (2016). Pre-slaughtering factors related to bruises on cattle carcasses. Animal Production Science, 58(2), 385-392.

Mendonça, F. S., Vaz, R. Z., Vaz, F. N., Restle, J., Gonçalves, G. B., \& Vara, C. C. (2017). Características raciais e de carcaça nas perdas por contusões e no $\mathrm{pH}$ final da carne de bovinos machos castrados e fêmeas de descarte. Ciência Animal Brasileira, 18(1), e45295.

Merchioratto, I., Monteiro, F. L., Traesel, C. K., Flores, E. F., Weiblen, R., \& Brum, M. (2020). Identification and characterization of pestiviruses isolated from individual fetal bovine serum samples originated in Rio Grande do Sul state, Brazil. Pesquisa Veterinária Brasileira, 40(5), $368-373$.

Mezzalira, J. C., Carvalho, P. C. D. F., Trindade, J. K. D., Bremm, C., Fonseca, L., Amaral, M. F. D., \& Reffatti, M. V. (2012). Produção animal e vegetal em pastagem nativa manejada sob diferentes ofertas de forragem por bovinos. Ciência Rural, 42(7), 1264-1270.

Missio, R. L., Restle, J., Moletta, J. L., Kuss, F., Neiva, J. N. M., \& Moura, I. C. F. (2013). Características da carcaça de vacas de descarte abatidas com diferentes pesos. Revista Ciência Agronômica, 44(3), 644-651.

Nielsen, S. S., Sandøe, P., Kjølsted, S. U., \& Agerholm, J. S. (2019). Slaughter of pregnant cattle in Denmark: Prevalence, gestational age, and reasons. Animals, 9(7), 392.

Njoga, U. J., Njoga, E. O., Nwobi, O. C., Abonyi, F. O., Edeh, H. O., Ajibo, F. E., Azor, N., Bello, A., Upadhyay, A. K., Okpala, C. O. R., Korzeniowska, M., \& Guiné, R. P. (2021). Slaughter Conditions and Slaughtering of Pregnant Cows in Southeast Nigeria: Implications to Meat Quality, Food Safety and Security. Foods, 10(6), 1298.

NRC - National Academies Of Sciences, Engineering, and Medicine et al. Nutrient requirements of beef cattle. 2016.

OIE - World Organization for Animal Health. Terrestrial Animal Health Code, Chapter 7.5, Article 7.5.5. 2016.

Oliveira, A., Tenedini, V., \& Ribeiro, L. F. (2021). Abate de vacas prenhas em abatedouros: bem-estar e soro fetal bovino. Revista GeTeC, 10(25), 14-23.

Pacheco, P. S., Restle, J., Missio, R. L., Menezes, L. F. G., Rosa, J. R. P., Kuss, F., Alves Filho, D. C., Neiva, J. N. M., \& Donicht, P. A. M. M. (2013). Características da carcaça e do corpo vazio de bovinos Charolês de diferentes categorias abatidos com similar grau de acabamento. Arquivo Brasileiro de Medicina Veterinária e Zootecnia, 65(1), 281-288.

Pascoal, L. L., Vaz, F. N., Vaz, R. Z., Restle, J., Pacheco, P. S., \& Santos, J. D. (2011). Relações comerciais entre produtor, indústria e varejo e as implicações na diferenciação e precificação de carne e produtos bovinos não-carcaça. Revista Brasileira de Zootecnia, 40 (Supl. Especial), $82-92$.

Pereira, A. S., Shitsuka, D. M., Parreira, F. J., \& Shitsuka, R. (2018). Metodologia da pesquisa científica. UFSM. https://repositorio.ufsm.br/bitstr eam/handle/1/15824/Lic_Computacao_Metodologia-Pesquisa-Cientifica.pdf?sequence=1.

Riehn, K., Domel, G., Einspanier, A., Gottschalk, J., Lochmann, G., Hildebrandt, G. \& Lücker, E. (2011). Schlachtung gravider Rinder-Aspekte der Ethik und des gesundheitlichen Verbraucherschutzes. Tierärztliche Umschau, 66 (1), 391-405.

Rio Grande do Sul (2020). Secretaria da Agricultura, Pecuária e Desenvolvimento Rural Departamento de Defesa Agropecuária Divisão de Defesa Sanitária Animal. Dados populacionais e de abate de bovinos no Estado do Rio Grande do Sul de 2015-2019. https://www.agricultura .rs.gov.br/upload/arquivos/202011/19101215-dados-populacionais-e-de-abate-de-bovinos-no-estado-do-rio-grande-do-sul-de-2015-2019.pdf

Silva, L. A. F., Pales, A. P., Fioravanti, M. C. S., Pádua, J. T., da Silva, O. C., \& dos Santos, K. J. G. (2006). Anel de látex aplicado no pedículo ovariano de bezerras Nelore. Acta Scientiarum. Animal Sciences, 28(1), 97-103.

Sornas, A. S., Júnior, P. R., \& Moizes, F. A. F. (2014). Impacto do abate de vacas prenhes sob parâmetros de carcaça e sua influência no resultado econômico. Archives of Veterinary Science, 19(4), 1-8.

Torres, H. A. L., Tineo, J. S. A., \& Raidan, F. S. S. (2015). Influência do escore de condição corporal na probabilidade de prenhez em bovinos de corte. Archivos de zootecnia, 64(247), 255-259.

Vaz, F. N., Vaz, R. Z., Pascoal, L. L., Pacheco, P. S., Miotto, F. R. C., \& Teixeira, N. P. (2012a). Análise econômica, rendimentos de carcaça e dos cortes comerciais de vacas de descarte 5/8 Hereford 3/8 Nelore abatidas em diferentes graus de acabamento. Ciência Animal Brasileira, 13(3), 338-345.

Vaz, F. N., Maysonnave, G. S., Pascoal, L. L., Vaz, R. Z., Severo, M. M., \& Fabrício, E. D. Á. (2021). Análise do valor agregado em bovinos certificados para o programa de carne angus no sul do Brasil. Ciência Animal Brasileira, 22(1), e57079.

Vaz, R. Z., da Silveira, M. F., Restle, J., Machado, D. S., da Silva, H. R., Garcia, J. A. B., \& da Conceição, V. G. D. (2020). Época de parto e produção de leite na eficiência bioeconômica de rebanhos de vacas de corte. Research, Society and Development, 9(9), e216997240.

Vaz, R. Z., Restle, J., Pacheco, P. S., Vaz, F. N., Pascoal, L. L., \& Vaz, M. B. (2012b). Ganho de peso pré e pós-desmame no desempenho reprodutivo de novilhas de corte aos quatorze meses de idade. Ciência Animal Brasileira, 13(3), 272-281.

Zago, D., Canozzi, M. E. A., \& Barcellos, J. O. J. (2019). Pregnant cow nutrition and its effects on foetal weight-a meta-analysis. The Journal of Agricultural Science, 157(1), 83-95.

Zitterer, I., \& Paulsen, P. (2021). Slaughter of Pregnant Cattle at an Austrian Abattoir: Prevalence and Gestational Age. Animals, $11(8), 2474$. 